\title{
VARIOUS APPLICATIONS OF AD-NET PRODUCTS FOR ENVIRONMENTAL IMPACT STUDIES RELATED TO ASIAN DUST
}

\author{
Atsushi Shimizu, Tomoaki Nishizawa, Yoshitaka Jin, Nobuo Sugimoto \\ National Institute for Environmental Studies, Tsukuba 305-8506 Japan \\ *Email:shimizua@nies.go.jp
}

\begin{abstract}
AD-Net, the Asian dust and aerosol lidar observation network, is operated in East Asian counties for creating useful dataset for environmental impact studies. The products from AD-Net have been utilized as the source of data assimilation in a chemical transport model, and as the index of exposure of Asian dust to human in the area. Long term observation is indispensable to estimate climatorogical change of dust density, and sustainable operation strategies are needed for further development of regional lidar network.
\end{abstract}

\section{INTRODUCTION}

In East Asia, mineral dust particles have been one of major target of lidar observations. Iwasaka et al.[1] reported the existence of aerosol layer at around 4-8 km over Nagoya, Japan using a lidar. Since then there were a lot of observational evidence of floating mineral dust with polarization lidars because the mineral dust particles have nonspherical shape which causes depolarized backscatter if lidars were employed to identify density and altitude of aerosol layer. The National Institute for Environmental Studies (NIES) has been operating dual wavelength polarization lidars in east Asian countries including Japan, Korea, China and Mongolia (Fig.1), and they are known as AD-Net (Asian dust and aerosol lidar network) which is a member of GALION (Gaw aerosol lidar observation network). For more than 15 years AD-Net has obtained vertical profiles of dust and spherical particle backscatter, and the product were utilized in various field of environmental impact studies. This paper exhibits the methodology of data production, examples of environmental impact studies, and future outlook of application of AD-Net.

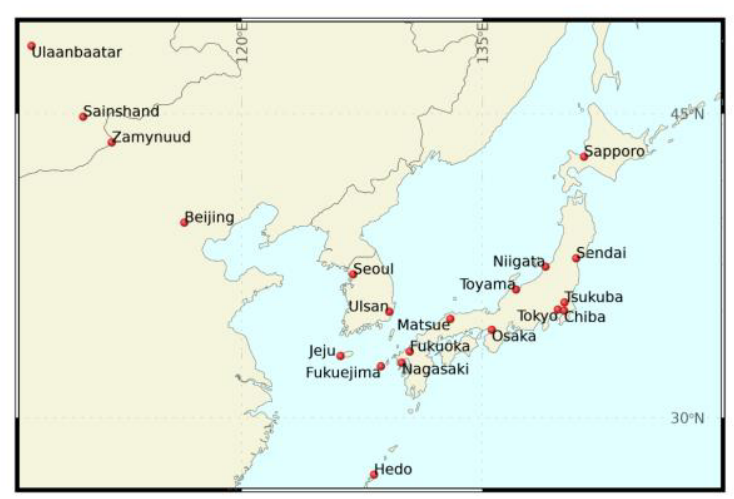

Fig.1 Locations of lidars in AD-Net.

\section{PRODUCTS FROM AD-NET}

Each AD-Net lidar is operated automatically in 24 hours regardless of whether conditions. Every 15 minutes the vertical profiles of three backscatter intensities, parallel / perpendicular components at $532 \mathrm{~nm}$ and total at $1064 \mathrm{~nm}$, are recorded with vertical resolution of 6 meters. Based on assumptions that lidar ratio (S1) is constant and two-components of particles (mineral dust and spherical particles) are externally mixed, 'dust extinction coefficient' and 'spherical particle coefficient' are calculated in semi real time[2]. Products in netCDF format and visualized figures are available online (http://wwwlidar.nies.go.jp/AD-Net/) except for data from stations in China. Two components of extinction coefficients are available with vertical resolution of $30 \mathrm{~m}$ below $9 \mathrm{~km}$ (in clear case) or below cloud base height, and the information related to cloud base / (apparent) top is also supplied. An example of visualized product is shown in Fig.2. 


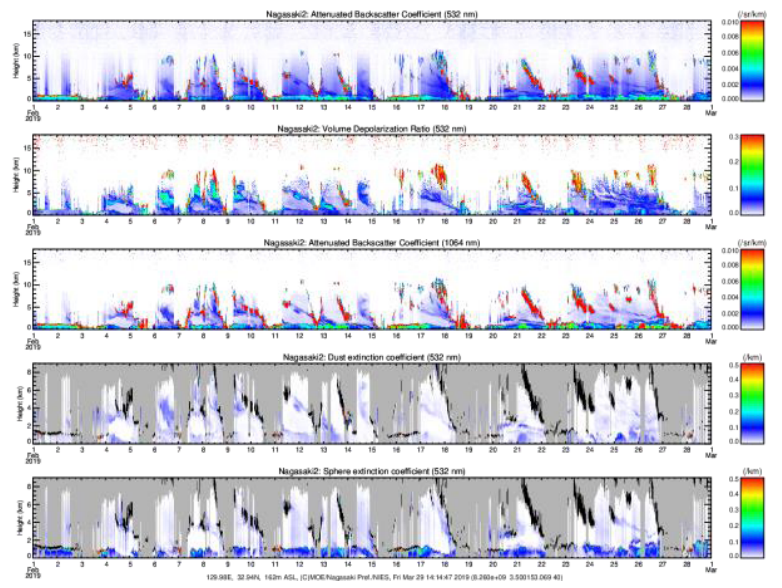

Fig.2 Time-height sections of [from top] attenuated backscatter coefficient (ABC) at 532 $\mathrm{nm}$, volume depolarization ration at $532 \mathrm{~nm}, \mathrm{ABC}$ at $1064 \mathrm{~nm}$, dust extinction coefficient with cloud mask, spherical particle extinction coefficient with cloud mask for February 2019 in Nagasaki, Japan.

\section{APPLICATIONS}

\subsection{Validation and data assimilation with numerical chemical transport models}

Validation of vertical distribution of particles estimated in chemical transport model (CTM) is one of traditional application of lidar observation, especially in Asian dust seasons[3]. Yumimoto et al.[4] firstly employed dust extinction coefficient as a source of data assimilation in 4D-VAR method. The effect of assimilation was confirmed by independent surface PM10 measurements in Asian dust event on March 2007. Re-analysis of aerosol distribution is under calculation by MRI [5] and dust extinction coefficient will be incorporated into it soon.

Recently Goto et al.[6] compared modeled vertical distribution of extinction coefficient by sulfate, carbonaceous and sea-salt with spherical particle extinction coefficient by lidars in AD-Net. Interpretation of spherical particle extinction coefficient is not straight like dust because it is affected by relative humidity of the ambient atmosphere that is not directly measured. Also, variations of lidar ratio (S1) in anthropogenic particles introduce the uncertainty of extinction coefficient. Thus, effective method to utilize spherical particle extinction coefficient with CTM is one big challenge of AD-Net.

\subsection{Epidemiology related to health impact of Asian dust}

Dust extinction coefficient near the surface is widely utilized as the index of mineral dust density because there is no other method to estimate the concentration of dust except for chemical analysis with filter sampling. Dust density is required in daily basis because health related data is usually daily, however simple averaging of dust extinction coefficient in 24 hours is sometimes contaminated by miss classification of cloud/rain. To avoid the problems, averaging of segmented medians, or correlation between dust extinction and SPM (suspended particulate matter, which is measured with betaray method at the surface) hourly data are examined. Several studies exhibited correlation between symptoms and Asian dust events in Japan [7-10].

\subsection{Climatology of Asian dust}

The occurrence of Asian dust at meteorological observatories in Japan made a peak around A.D.2000 and recently it decreases gradually. However the quantitative assessment based on daily SYNOP report is not easy. Thus, 'Dust $A D^{\prime}(t a u$ d) is defined from dust extinction coefficient and annual cycle of tau d was fitted to the combination of linear function and sinusoidal curve with a period of one year [11]. As a result, tau_d decreased $-2.5 \%$ per year over Japan (Fig.3), and this trend was more significant in upper region $(5-6 \mathrm{~km})$. This trend was not clear in Mongolian region, which suggests that the negative trend of Asian dust over Japan is not directly related to the change of generation of Asian dust in source region but to the change of transportation route to Japan/northwestern Pacific region. Another challenge is discrimination of long-range transported Asian dust and locally generated dust in Japan. In early spring dust particles are blown from crop fields in Japan, and it causes highly depolarized backscatter. Although current algorithm of AD-Net cannot distinguish such local dust and Asian dust, optical properties must be different among these two kinds of dust. More sophisticated algorithm is required to 
identify the climatological change of Asian dust transport.

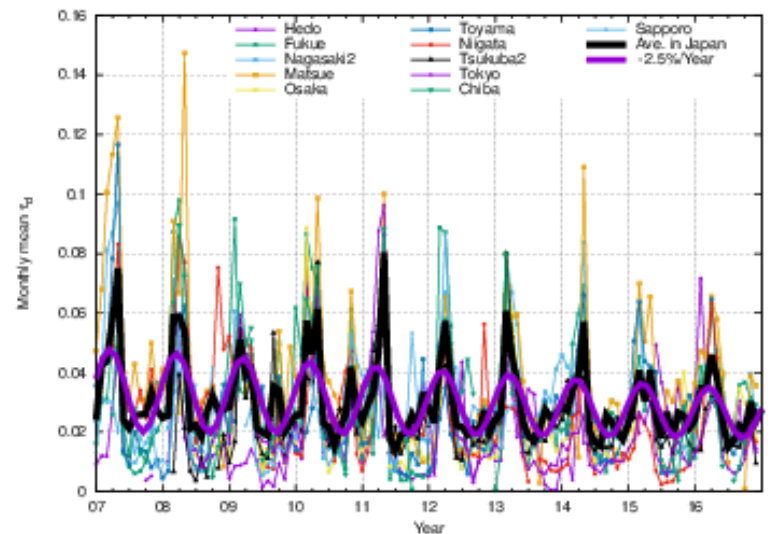

Fig.3 Monthly variations of Tau d since 2008 observed by independent lidars in Japan (thin lines), average in Japan (thick line) and fitted curve to average (solid purple). Reproduced from [11].

\subsection{Contribution to International Activities}

Products of AD-Net are also utilized in the international collaborative activities. In Dust and Sand Storm Working Group 1 (DSS-WG1) under Tripartite Environmental Ministry Meeting (TEMM), Japan, Korea and China exchange observational results during specified Asian dust events, and data of AD-Net is important resource to identify such events. AD-Net is also expected to supply products to WMO Sand and Dust Storm Warning Advisory and Assessment System (SDSWAS).

\section{FUTURE OUTLOOK}

Although rapid economic growth in East Asia caused significant enhancement of anthropogenic aerosols in past few decades, the emission of precursors of aerosols are now decreasing. Thus the natural particles like Asian dust becomes relatively more important to evaluate atmospheric conditions. To identify the shift of dust emission area, or the main transport path of Asian dust to western Pacific region, observational evidence by AD-Net and CTM must be combined more closer. Once reanalysis data set made by CTM becomes available, health impact studies are possible even in the region where lidar is not operated. Additionally hemispheric transport of mineral dust, namely Saharan/Arabian dust transport to East Asia is a new topic in observational studies. To catch them, lidars must be located in wider area in the region. To maintain the existing lidar stations and to identify the effective locations of (possible) new stations are important to sustain AD-Net activities.

\section{ACKNOWLEDGEMENTS}

Operation of AD-Net is supported by The Ministry of Environment Japan, and Environmental Research and Technology Development Fund (E-1502) by Environmental Restoration and Conservation Agency, Japan.

\section{REFERENCES}

[1]Iwasaka et al., Tellus 35B:189-196 (1983)

[2]Shimizu et al., Opt. Eng. 56(3):031219 (2016)

[3]Shimizu et al., J. Geohpys. Res. 109:D19S17 (2004)

[4]Yumimoto et al., Atmos. Chem. Phys. 8(11):28692884 (2008)

[5]Yumimoto et al., Geosci. Model Dev. 10:3225-3253 (2017)

[6]Goto et al., J. Geophys. Res. 120:6247-6270 (2015)

[7]Ueda et al., Toxicology 24(12):858-867 (2012)

[8]Higashi et al., J. Atmos. Env. 97:537-543 (2014)

[9]Kanatani et al., Annals. Allergy Asthma Immunology 116(5):425-430 (2016)

[10]Nakamura et al., J. Epidemiology 26(11):593-601 (2016)

[11]Shimizu et al., Sci. Online Let. Atmos. 13:205-208 (2017) 